\title{
Nanoparticles Containing Hyaluronan Acid and Astragalus Polysaccharides for Treating Osteoarthritis
}

\author{
Gongbiao Lu, ${ }^{1}$ Lin Du, ${ }^{2}$ Jishou Lu, ${ }^{1}$ and Liuzhong Jin $\mathbb{D}^{1}$ \\ ${ }^{1}$ Department of Spine Surgery, Jining No. 1 People's Hospital, Jining, Shandong 272011, China \\ ${ }^{2}$ School of Nursing, Jining Medical University, Jining 272013, China \\ Correspondence should be addressed to Liuzhong Jin; 13455580113@163.com
}

Received 18 March 2019; Revised 21 May 2019; Accepted 28 June 2019; Published 14 July 2019

Guest Editor: Di Li

Copyright (C) 2019 Gongbiao Lu et al. This is an open access article distributed under the Creative Commons Attribution License, which permits unrestricted use, distribution, and reproduction in any medium, provided the original work is properly cited.

\begin{abstract}
The pathogeny of osteoarthritis (OA) is very complicated and still is one of the difficulties in a treating procedure. Here, we constructed nanoparticles using hyaluronan acid (HA) and astragalus polysaccharides (APS) for OA therapy. We assessed OA biomarkers and IL-1 $\beta$-induced matrix metalloproteinase (MMP) expressions. Nanoparticles of $100 \mathrm{~nm}$ showed high drug loading of $28.6 \%(w / w)$ and extended drug release of $59 \%$ over 1 month. Our results demonstrated that nano treatment significantly improved IL- $1 \beta$-induced cell viability of chondrocytes. Induction of MMP-9, MMP-13, and TNF- $\alpha$ was alleviated by nanoparticles. Furthermore, nano elevated the expression of osteopontin (OPN) and attenuated inducible nitric oxide synthase (iNOS) protein. Our data indicated the protective role of HA and APS-capsuled nanoparticles in OA treatment.
\end{abstract}

\section{Introduction}

Polysaccharides not only are the main constituent of natural products and energy suppliers but also have exclusive and important biological activities. For example, it can improve the immunity and has obvious antioxidation and anticoagulation effects [1]. These bioactivities of polysaccharides are important for organisms, which have become the focus of many research disciplines. As reported, many traditional Chinese medicine polysaccharides used alone or combined with other traditional Chinese medicine polysaccharides can not only improve the local microcirculation but also reduce the microcirculation intraosseous pressure, scavenge oxygen free radicals, and inhibit synovitis and cartilage apoptosis and abnormal regulation of cytokines [2-5]. The pathological changes of the immune organs can improve the synovial lesion of the joint and effectively inhibit the inflammation. Therefore, the research on astragalus polysaccharides (APS) of traditional Chinese medicine may become one of the important directions of prevention and cure of OA.

Accumulated evidence has revealed the potential mechanism of prevention and treatment of $\mathrm{OA}$ by polysaccharide of traditional Chinese medicine [6]. To tackle the dilemma of rapid clearance of biologically active molecules from the lesion, nanoparticles were considered in this study. HA is also a type of biopolymer widely used in treating OA. However, injected HA is not a long-lasting treatment and remains uncertain because of the degradation [7]. Polypeptide micelles combined with antitumor drug and polyion complex have been documented $[8,9]$. In addition, recently, HA has been used in drug delivery nanoparticles for targeting cell-surface glycoprotein 44- (CD44-) overexpressed solid tumors [10]. Considering that HA helps the elasticity of synovial fluid and binds to CD44 which is highly expressed in chondrocytes, we used HA as a drug carrier and fabricated HA-coated nanoparticle to target OA sites.

Thus, in the present study, we developed a novel drug delivery system composed of hyaluronan acid and astragalus polysaccharides. We subsequently evaluated the therapeutic effects of formulated HA and APS-capsuled nanoparticles on isolated human articular chondrocytes.

\section{Materials and Methods}

2.1. Synthesis of Nanoparticles. The nanoparticles formulated are comprised of two polymers: HA and APS. The 
preparation of nanoparticles was performed as described previously [11]. We followed and modified the methods of Maudens et al. [12]. 1,2-Dilauroyl-sn-glycero-3-phosphoethanolamine (DLPE) and 1,2-dilauroyl-sn-glycero-3glycerol (DLPG) were from Avanti Polar Lipids Inc. (Alabaster, AL, USA). HA obtained from Sigma-Aldrich (St. Louis, MO, USA) was diluted to $2 \mathrm{mg} / \mathrm{mL}$ in distilled water. APS and lipids (DLPE:DLPG mole ratio of 7:3) were dissolved in ethanol and mixed with HA. Nanoparticle solution was evaporated to dry overnight at room temperature in a Buchi Rotary Evaporator Vacuum System. Nanoparticle size was calculated by dynamic light scattering (DLS) using a BI-200SM size analyzer. The nanosuspensions were lyophilized and stored at $4^{\circ} \mathrm{C}$ for further use. The concentration of nano formulation includes $8 \%$ ethanol (96\%), 92.8\% HA $(\mathrm{mL} / \mathrm{mL})$, and $0.2 \%$ APS $(\mathrm{g} / \mathrm{mL})$.

2.2. In Vitro Drug Release. Saline solution $(0.9 \%(w / v) \mathrm{NaCl})$ and nano were saturated with the dexamethasone base $(0.05$, 0.50 , and $2.50 \mathrm{mg} \mathrm{mL}^{-1}$, respectively) at $20^{\circ} \mathrm{C}$. Ten milliliters of each of the previous solutions was dialyzed (MWCO: 1000 , Spectra/Por ${ }^{\circledR}$ membrane) against $400 \mathrm{~mL}$ of $0.9 \%$ $(w / v) \mathrm{NaCl}$ for $28 \mathrm{~h}$ at $37^{\circ} \mathrm{C}$ and $80 \mathrm{RPM}$. The polymer matrix was hydrolyzed by heating $\left(121^{\circ} \mathrm{C}\right)$ to release the remaining content of dexamethasone, and DMSO was added (1:1, water:DMSO). The quantity of the drug released at each time point was quantified by reversed-phase UHPLC using a C18 Hypersil Gold column (50/2.1, $1.9 \mu \mathrm{m}$ bead particle size, Thermo Scientific, Waltham, USA). The mobile phase consists of $0.1 \%(v / v)$ formic acid in water (A) and $0.1 \%$ $(v / v)$ trifluoroacetic acid (TFA) in acetonitrile (B), and the following gradient elution sequence was applied at a flow rate of $400 \mu \mathrm{L} \mathrm{min}$ m $^{-1}$ 30-95\% A (0-3 min), 95-10\% A (3-4 min), $10-30 \% \mathrm{~A}(4-4.5 \mathrm{~min})$, and $30 \% \mathrm{~A}$ (4.5-5 min).

2.3. Isolation of Articular Chondrocytes. OA model rabbits (New Zealand female rabbits, 8-month-old, $4.5 \pm 0.5 \mathrm{~kg}$ ) were created using the classic Hulth method as previously reported [2]. Synovial tissues were isolated from the knee joints of New Zealand white rabbits (female, average weight: $2.5 \pm 0.5 \mathrm{~kg}$ ) after being sacrificed with overdose of pentobarbital sodium (Sigma-Aldrich) under sterile conditions. The experimental protocol was approved by the Institution's Animal Care and Use Committee of Jining No. 1 People's Hospital. Cartilage was cut into small pieces and digested by $0.2 \%$ collagenase II (Gibco, Carlsbad, CA, USA) at $37^{\circ} \mathrm{C}$ for $12 \mathrm{~h}$. After washing twice with PBS, the isolated synoviocytes were cultured in monolayers in culture medium composed of DMEM/F12 (Gibco) supplemented with 10\% fetal bovine serum (FBS; Gibco, USA) and $1 \%$ antibiotics at $37^{\circ} \mathrm{C}$ with $5 \% \mathrm{CO}_{2}$.

2.4. Release of Nanoparticles. Lyophilized nanoparticle powder $(2 \mathrm{mg})$ was resuspended with $5 \mathrm{~mL}$ phosphate-buffered solution (PBS) and shaken at $100 \mathrm{r} / \mathrm{min}$ at $37^{\circ} \mathrm{C}$. $500 \mu \mathrm{L}$ of buffer was collected and measured at a predetermined time point. The amounts of releases were calculated as follows: accumulated released ration $=$ APS amount in buffer/ total APS amount $\times 100 \%$. The encapsulation efficiency

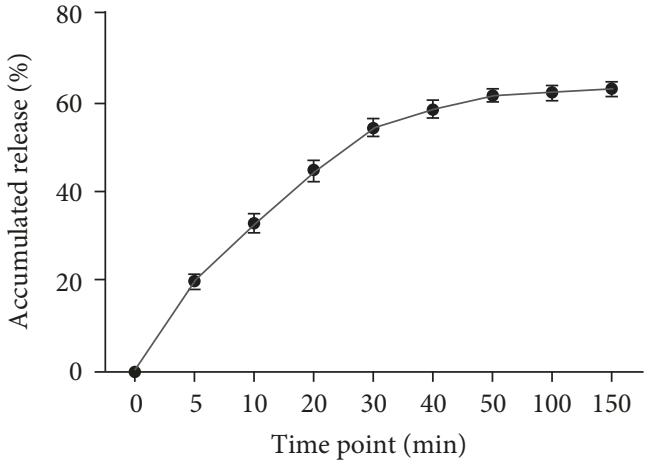

FIGURE 1: Release of nanoparticles in PBS (pH 7.4). Data are expressed as the mean \pm standard deviation (SD) $(n=4)$. At least three independent experiments were conducted.

(EE) and loading efficiency (LE) were determined as previously reported [13]: drug loading $=$ wt drug entrapped/wt microparticles $\times 100 \%$.

2.5. Cytotoxicity Assays. Cell proliferation was assayed using the Cell Counting Kit-8 (CCK-8; Dojindo, Japan). $100 \mathrm{~L}$ cell $\left(2 \times 10^{6} /\right.$ well $)$ suspension was prepared in a 96 -well plate $\left(37^{\circ} \mathrm{C}\right.$ and $5 \% \mathrm{CO}_{2}$ ) for 24 hours. Various concentrations of nanoparticles $(0.5,5,10$, and $100 \mathrm{mg} / \mathrm{L}$ APS $)$ or IL- $1 \beta$ $(10 \mathrm{ng} / \mathrm{mL})$ were added to each well at different concentrations and incubated for 24 hours. There were 6 groups in the present study: control (DMEM medium), IL- $1 \beta$, IL$1 \beta+0.5 \mathrm{mg} / \mathrm{L}$ APS, IL- $1 \beta+5 \mathrm{mg} / \mathrm{L}$ APS, IL- $1 \beta+10 \mathrm{mg} / \mathrm{L}$ APS, and IL- $1 \beta+100 \mathrm{mg} / \mathrm{L}$ APS. After incubation for $24 \mathrm{~h}$, add $10 \mathrm{~L}$ CCK-8 solution to each well. Incubate the plates in the incubator for 1-4 hours. The absorbance at $450 \mathrm{~nm}$ was determined by a microplate analyzer (model 680; Bio-Rad, Hercules, CA, USA). Chondrocytes cultured without NMPs served as the control group.

2.6. Measurement of $I L-1 \beta$, iNOS, OPN, and TNF- $\alpha$. Blood samples collected from rabbit were centrifuged at $1000 \mathrm{~g}$ for $15 \mathrm{~min}$ and $10000 \mathrm{~g}$ for $10 \mathrm{~min}$ at $4^{\circ} \mathrm{C}$. OPN, iNOS, and TNF- $\alpha$ were measured by ELISA (Hercules, CA, USA) according to the specifications of the manufacturer. Data were analyzed by Bio-Plex Manager software 6.1 to obtain concentrations.

2.7. Western Blot Analysis. Proteins were extracted from the articular chondrocytes. After the concentrations of the proteins were evaluated, equal amounts of protein were electrophoresed on sodium dodecyl sulfate-polyacrylamide gel and transferred to polyvinylidene difluoride (PVDF) membranes. The membranes were blocked with $2 \%$ nonfat dry milk in Tris-buffered saline-0.05\% Tween ( $\mathrm{pH} 7.4$ ) and incubated with anti-OPN and anti-iNOS antibodies $(1: 2000$, Abcam) at $4^{\circ} \mathrm{C}$ overnight. The membrane was washed three times and incubated with horseradish peroxidase-conjugated secondary antibody (Abcam) at $37^{\circ} \mathrm{C}$ for $2 \mathrm{~h}$. The protein bands were visualized by an enhanced chemiluminescence system, and GAPDH (Abcam) was used as an internal control to normalize. 


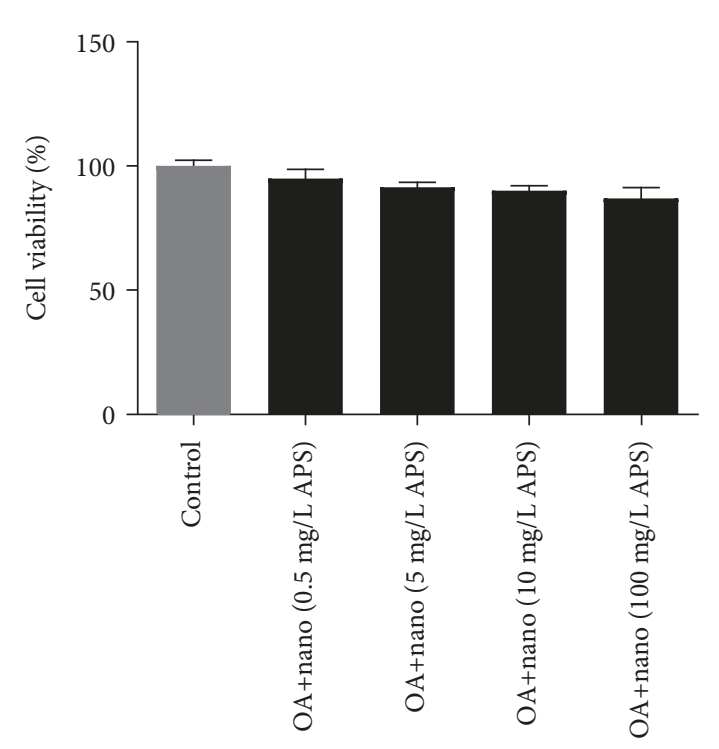

(a)

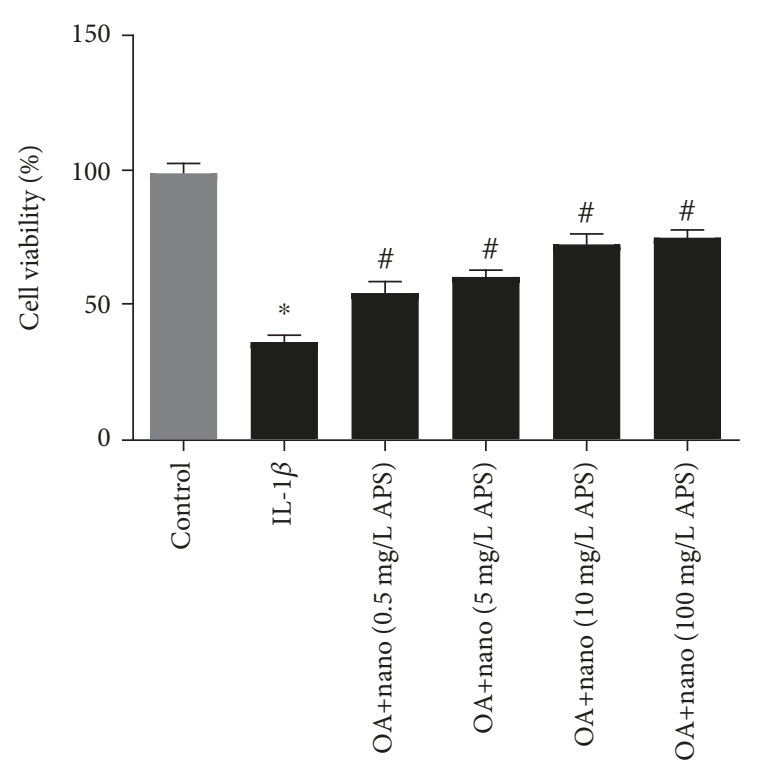

(b)

Figure 2: Nanoparticles attenuate the inhibitory effect on the cell viability induced by IL- $1 \beta$. Cell viability of articular chondrocytes was evaluated using the CCK- 8 assay. At least three independent experiments were conducted. (a) OA chondrocytes were treated with various concentrations of APS-loaded nanoparticles $(0,0.5,5,10$, and $100 \mathrm{mg} / \mathrm{L})$ for $24 \mathrm{~h}$. (b) OA chondrocytes were treated with various concentrations of APS-loaded nanoparticles $(0.5,5,10$, and $100 \mathrm{mg} / \mathrm{L})$ with $\mathrm{IL}-1 \beta(10 \mathrm{ng} / \mathrm{mL})$ for $24 \mathrm{~h} .{ }^{*} P<0.05$ compared with the control group, ${ }^{\#} P<0.05$ compared with the IL- $1 \beta$ group.

2.8. Statistical Analysis. All the continuous data were presented as the mean \pm SD. All statistical analysis was performed using SPSS17.0. Unpaired Student's $t$-test was used for the comparisons between two different groups. $P<0.05$ was considered significant.

\section{Results}

3.1. Basic Characteristics of APS-Loaded Nanoparticles. The nanoparticles have an average diameter of $110.6 \pm 12.8 \mathrm{~nm}$. The encapsulation efficiency was $32.4 \pm 0.19 \%$, and the loading efficiency was $75.0 \pm 0.02 \%$. The APS was released from nanoparticles in PBS (Figure 1). With the degradation of $\mathrm{HA}$, the structure of nanoparticles was broken up and APS was released. In addition, nanoparticles of $100 \mathrm{~nm}$ showed high drug loading of $28.6 \%(w / w)$ and extended drug release of $59 \%$ over 1 month.

3.2. Cytotoxicity of Nanoparticles. We next examined the effect of nanoparticles on cell viability using the CCK-8 assay. As shown in Figure 2(a), treatment with nanoparticles caused slight decrease in cell viability. Also, we observed that when compared with the control group, IL- $1 \beta$ treatment remarkably decreased the cell viability of articular chondrocytes (Figure 2(b), $P<0.05$ ). However, APS-capsuled nanoparticles sharply improved cell viability inducted by IL- $1 \beta$ in a dose-dependent manner. There is no significant difference between 10 and $100 \mathrm{mg} / \mathrm{L}$ APS; thus, $0.5-10 \mathrm{mg} / \mathrm{L}$ of APS was used in the following experiments.

3.3. The Production of MMP-9, MMP-13, and TNF- $\alpha$. ELISA results showed that nano containing $0.5,5$, and $10 \mathrm{mg} / \mathrm{L}$ APS significantly decreased the concentrations of MMP-9, MMP13 , and TNF- $\alpha$, which was significantly elevated by IL- $1 \beta$ (Figure 3, $P<0.05$ ). These results indicated that APSloaded nano could reduce the levels of inflammation cytokines and matrix-degrading proteins, therefore protecting articular chondrocytes.

3.4. The Expression of OPN and iNOS. Then, we verified the effects of APS on OPN and iNOS in OA chondrocytes cultured with IL- $1 \beta$. The data of the Western blot assay demonstrated that IL- $1 \beta$ significantly decreased the protein expression of OPN and increased the expression of iNOS, as compared with the control (Figure 4). However, APS-encapsuled nano markedly elevated IL- $1 \beta$ induced OPN proteins and attenuated IL- $1 \beta$-induced iNOS protein expression.

\section{Discussion}

All over the world, osteoarthritis is the most common joint disease, disturbing approximately $10 \%$ of men and $18 \%$ of women aged over 60 , which was regarded as immortal cancer [14]. In general, there are various aspects in the pathogenesis process, including cytokines, apoptosis, and protease [15]. Current therapies are not effective or have unwanted side effects. Self-targetability has emerged as a new targeting strategy that has been used in breast tumor, orthotopic hepatoma, and other diseases [16, 17]. Recently, a body of evidence showed that polysaccharide drugs for $\mathrm{OA}$ are a promising approach in early and midterm treatment [18]. APS has been used in the treatment of OA in the past years. In this study, APS was chosen as a therapeutic agent 


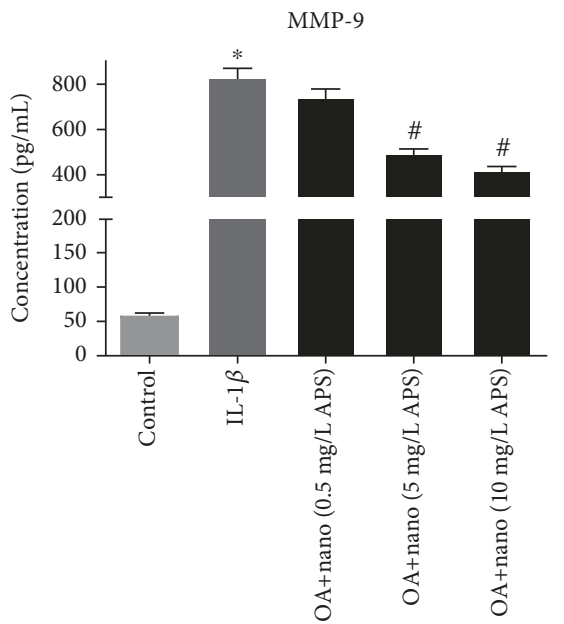

(a)

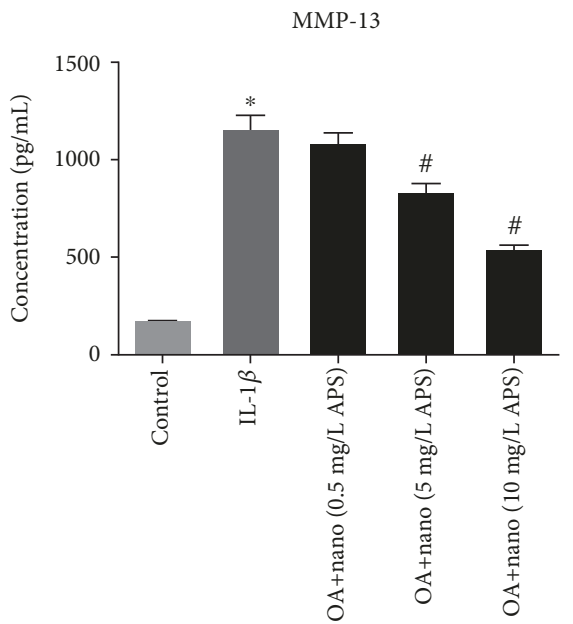

(b)

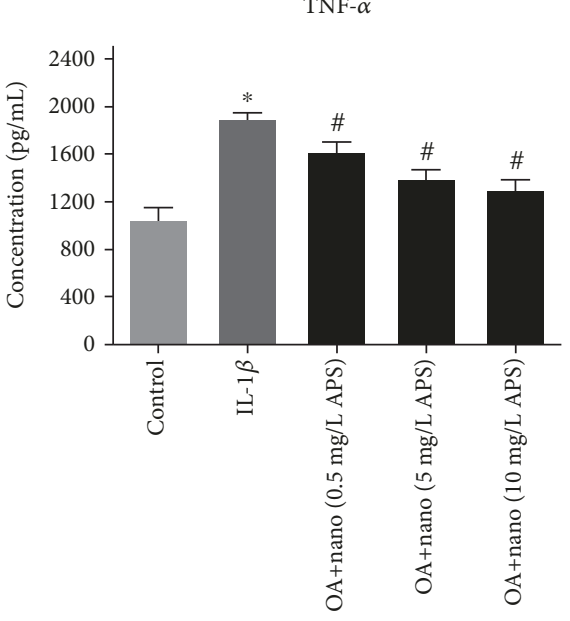

(c)

Figure 3: The levels of MMP-9, MMP-13, and TNF- $\alpha$ were determined using ELISA. The values are expressed as the mean \pm standard deviation (SD). ${ }^{*} P<0.05$ compared with the control group; ${ }^{\#} P<0.05$ compared with the IL- $1 \beta$ group.
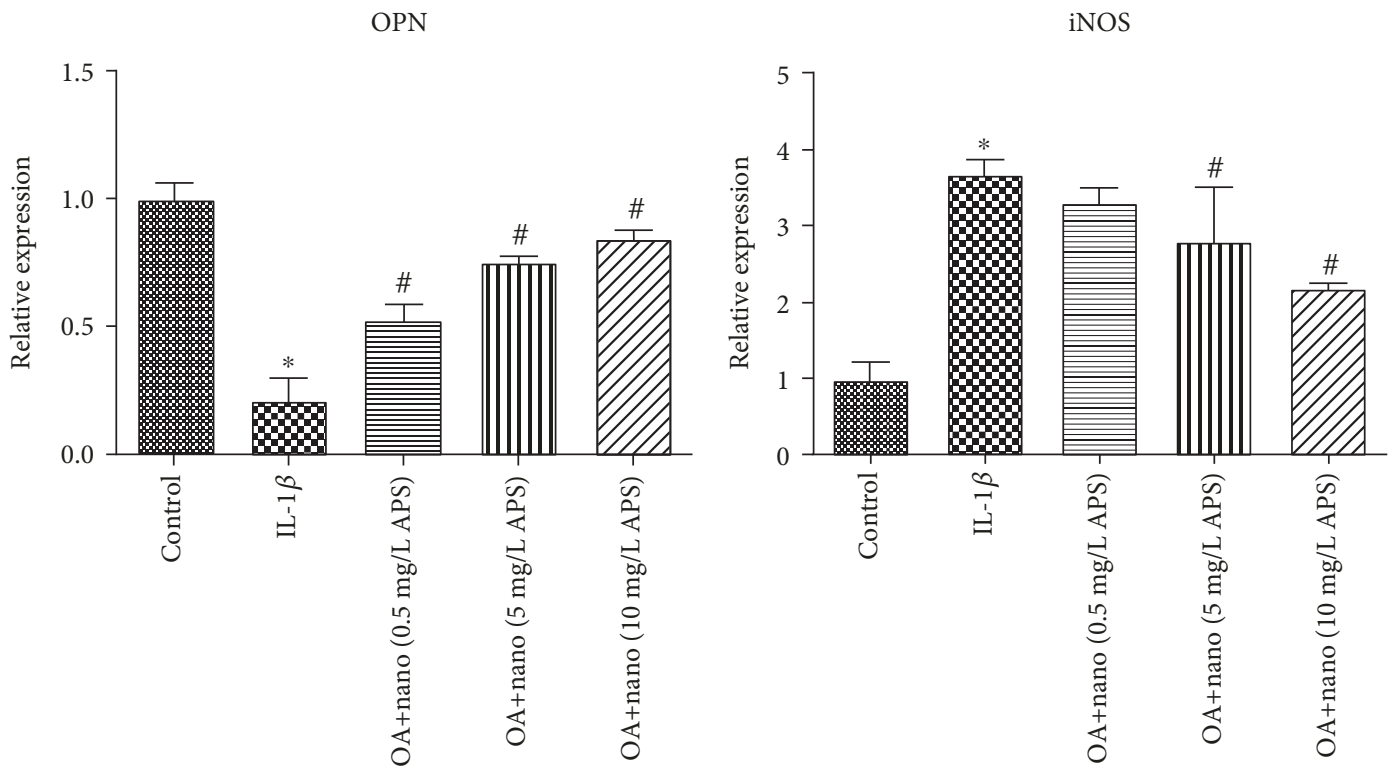

FIGURE 4: The protein levels of OPN and iNOS in articular chondrocytes. OA chondrocytes were pretreated with various concentrations of APS-loaded nanoparticles. ${ }^{*} P<0.05$ compared with the control group; ${ }^{*} P<0.05$ compared with the IL- $1 \beta$ group. OPN: osteopontin.

because of the specific biological effect of polysaccharides. Hydrogels composed of natural polymers such as hyaluronic acid have been used in the delivery of biologies. Importantly, HA is capable of enhancing delivery efficiency for chondrocytes due to the fact that CD44 is the primary receptor for HA internalization, thus facilitating the access to chondrocytes in the joint. Due to the small size and large specific surface area, nanoparticles are considered to be a promising medicine $[5,19,20]$. In the present study, we first combined these two agents and encapsulated both APS and HA in the nano structure.

In our study, the APS-loaded nano were prepared successfully (the morphology of microspheres was not shown). The data of CCK- 8 assays showed that various concentrations of nanoparticles presented low cytotoxicity and improved the IL- $1 \beta$-induced damage in chondrocytes. Based on the cytotoxicity data, we believed that the nano was safe and excellent.

It has been known that the MMP family is a vital biomarker related to articular cartilage degeneration [21]. MMPs are a big family including MMP-1, MMP-2, MMP3, MMP-5, MMP-9, and MMP-13 [22]. Among these, MMP-9 and MMP-13 are well-studied in the role of OA. Elevation of MMP-9 and MMP-13 is responsible for cleaving extracellular matrix and the construction of chondrocytes [23-25]. Here, we found that our modified nano declined the elevation of MMP-9 and MMP-13 induced by IL-1 $\beta$. Moreover, the nano had significantly decreased the expression of inflammatory factor TNF- $\alpha$, indicating the anti-inflammation role of APS and HA combined nano. 
OPN is known as a cytokine related to OA metabolism; it is noncollagen bone matrix glycoprotein that mediates varied kinds of biological behaviors and often presents in the cartilage and synovial fluid of patients with OA [26]. OPN is an inhibitor to inflammatory substances such as IL-1 and NO, thus inhibiting the inflammatory process in cartilage [27]. iNOS is an isoform of the NO synthase family of enzymes, which correlates with the development of toxicity [28]. Stimulation of IL-1 $\beta$ could increase the expression of iNOS in chondrocytes therefore promoting NO production [29]. We investigated the effect of nanosphere on the production of OPN and iNOS. Western blot analyses indicated that the nanosphere effectively attenuated the levels of OPN and iNOS, leading to suppression of the inflammatory process. These results suggested that the anti-inflammatory mechanism of nanosphere is due to the inhibition of key cytokines.

In summary, we provide a new microsphere of modified medicine that overcomes the quick degradation of drugs. We conclude that APS- and HA-loaded nano can effectively promote the survival of chondrocytes and protect chondrocytes by regulating the cytokines and proteinase.

\section{Data Availability}

All the data is available with the handwritten notebook documented in our lab.

\section{Conflicts of Interest}

The authors declare that they have no conflicts of interest.

\section{Authors' Contributions}

Gongbiao Lu and Lin Du contributed equally to this work.

\section{References}

[1] P. Rozi, A. Abuduwaili, P. Mutailifu et al., "Sequential extraction, characterization and antioxidant activity of polysaccharides from Fritillaria pallidiflora Schrenk," International Journal of Biological Macromolecules, vol. 131, pp. 97-106, 2019.

[2] J. N. Rogart, H. J. Barrach, and C. O. Chichester, "Articular collagen degradation in the Hulth-Telhag model of osteoarthritis," Osteoarthritis and Cartilage, vol. 7, no. 6, pp. 539547, 1999.

[3] Z. Liang, Z. Yuan, J. Guo et al., "Ganoderma lucidum polysaccharides prevent palmitic acid-evoked apoptosis and autophagy in intestinal porcine epithelial cell line via restoration of mitochondrial function and regulation of MAPK and AMPK/Akt/mTOR signaling pathway," International Journal of Molecular Sciences, vol. 20, no. 3, p. 478, 2019.

[4] N. Yu, N. Song, C. Liu, and G. Yang, "The estrogen-like protective effect of Lycium barbarum polysaccharides in reducing oxidative stress on myocardial cells from ovariectomized rats," Molecular Medicine Reports, vol. 19, no. 3, pp. 2271-2278, 2019.

[5] L. J. Ke, G. Z. Gao, Y. Shen, J. W. Zhou, and P. F. Rao, "Encapsulation of aconitine in self-assembled licorice protein nanoparticles reduces the toxicity in vivo," Nanoscale Research Letters, vol. 10, no. 1, p. 449, 2015.
[6] Q. Chen, X. Shao, P. Ling, F. Liu, G. Han, and F. Wang, "Recent advances in polysaccharides for osteoarthritis therapy," European Journal of Medicinal Chemistry, vol. 139, pp. 926-935, 2017.

[7] A. Avenoso, A. D'Ascola, M. Scuruchi et al., "Hyaluronan in experimental injured/inflamed cartilage: in vivo studies," Life Sciences, vol. 193, pp. 132-140, 2018.

[8] W. Xu, J. Ding, and X. Chen, "Reduction-responsive polypeptide micelles for intracellular delivery of antineoplastic agent," Biomacromolecules, vol. 18, no. 10, pp. 3291-3301, 2017.

[9] J. Wang, W. Xu, H. Guo et al., "Selective intracellular drug delivery from $\mathrm{pH}$-responsive polyion complex micelle for enhanced malignancy suppression in vivo," Colloids and Surfaces B: Biointerfaces, vol. 135, pp. 283-290, 2015.

[10] C. Yang, Y. He, H. Zhang et al., "Selective killing of breast cancer cells expressing activated CD44 using CD44 ligand-coated nanoparticles in vitro and in vivo," Oncotarget, vol. 6, no. 17, pp. 15283-15296, 2015.

[11] G. Bachar, K. Cohen, R. Hod et al., "Hyaluronan-grafted particle clusters loaded with mitomycin $\mathrm{C}$ as selective nanovectors for primary head and neck cancers," Biomaterials, vol. 32, no. 21, pp. 4840-4848, 2011.

[12] P. Maudens, S. Meyer, C. A. Seemayer, O. Jordan, and E. Allémann, "Self-assembled thermoresponsive nanostructures of hyaluronic acid conjugates for osteoarthritis therapy," Nanoscale, vol. 10, no. 4, pp. 1845-1854, 2018.

[13] Z. Chen, S. Deng, D. C. Yuan et al., "Novel nano-microspheres containing chitosan, hyaluronic acid, and chondroitin sulfate deliver growth and differentiation factor-5 plasmid for osteoarthritis gene therapy," Journal of Zhejiang University-Science $B$, vol. 19, no. 12, pp. 910-923, 2018.

[14] A. D. Woolf and B. Pfleger, "Burden of major musculoskeletal conditions," Bulletin of the World Health Organization, vol. 81, no. 9, pp. 646-656, 2003.

[15] D. Chen, J. Shen, W. Zhao et al., "Osteoarthritis: toward a comprehensive understanding of pathological mechanism," Bone Research, vol. 5, no. 1, article 16044, 2017.

[16] C. Yang, Y. Liu, Y. He et al., "The use of HA oligosaccharideloaded nanoparticles to breach the endogenous hyaluronan glycocalyx for breast cancer therapy," Biomaterials, vol. 34, no. 28, pp. 6829-6838, 2013.

[17] D. Li, W. Xu, P. Li et al., "Self-targeted polysaccharide prodrug suppresses orthotopic hepatoma," Molecular Pharmaceutics, vol. 13, no. 12, pp. 4231-4235, 2016.

[18] M. M.-G. Sun, F. Beier, and M. A. Pest, "Recent developments in emerging therapeutic targets of osteoarthritis," Current Opinion in Rheumatology, vol. 29, no. 1, pp. 96-102, 2017.

[19] H. Guo, F. Li, W. Xu et al., "Mucoadhesive cationic polypeptide nanogel with enhanced penetration for efficient intravesical chemotherapy of bladder cancer," Advanced Science, vol. 5, no. 6, article 1800004, 2018.

[20] J. Wang, W. Xu, S. Li et al., "Polylactide-cholesterol stereocomplex micelle encapsulating chemotherapeutic agent for improved antitumor efficacy and safety," Journal of Biomedical Nanotechnology, vol. 14, no. 12, pp. 2102-2113, 2018.

[21] C. J. Malemud, "Matrix metalloproteinases and synovial joint pathology," Progress in Molecular Biology and Translational Science, vol. 148, pp. 305-325, 2017.

[22] N. Cui, M. Hu, and R. A. Khalil, "Biochemical and biological attributes of matrix metalloproteinases," Progress in Molecular Biology and Translational Science, vol. 147, pp. 1-73, 2017. 
[23] A. Bahtiar, M. Nurazizah, T. Roselina, A. P. Tambunan, and A. Arsianti, "Ethanolic extracts of babandotan leaves (Ageratum conyzoides L.) prevents inflammation and proteoglycan degradation by inhibiting TNF- $\alpha$ and MMP-9 on osteoarthritis rats induced by monosodium iodoacetate," Asian Pacific Journal of Tropical Medicine, vol. 10, no. 3, pp. 270-277, 2017.

[24] H. Li, D. Wang, Y. Yuan, and J. Min, "New insights on the MMP-13 regulatory network in the pathogenesis of early osteoarthritis," Arthritis Research \& Therapy, vol. 19, no. 1, p. 248, 2017.

[25] J. Song, E. H. Jin, D. Kim, K. Y. Kim, C. H. Chun, and E. J. Jin, "MicroRNA-222 regulates MMP-13 via targeting HDAC-4 during osteoarthritis pathogenesis," BBA Clinical, vol. 3, pp. 79-89, 2015.

[26] Z. Bai, X. H. Guo, C. Tang, S. T. Yue, L. Shi, and B. Qiang, "Effects of artesunate on the expressions of insulin-like growth factor-1, osteopontin and C-telopeptides of type II collagen in a rat model of osteoarthritis," Pharmacology, vol. 101, no. 1-2, pp. 1-8, 2018.

[27] M. G. Attur, M. N. Dave, S. Stuchin et al., "Osteopontin: an intrinsic inhibitor of inflammation in cartilage," Arthritis \& Rheumatism, vol. 44, no. 3, pp. 578-584, 2001.

[28] Z. Kucuktag, B. Satar, S. Yetiser, Y. Hidir, and O. Gunhan, "Immunohistochemical investigation of inducible nitric oxide synthase, osteopontin, and calcium-sensing receptor in a myringosclerosis/tympanosclerosis model," Otology \& Neurotology, vol. 35, no. 1, pp. e15-e23, 2014.

[29] Z. Ma, Y. Wang, T. Piao, and J. Liu, "Echinocystic acid inhibits IL- $1 \beta$-induced COX- 2 and iNOS expression in human osteoarthritis chondrocytes," Inflammation, vol. 39, no. 2, pp. 543-549, 2016. 


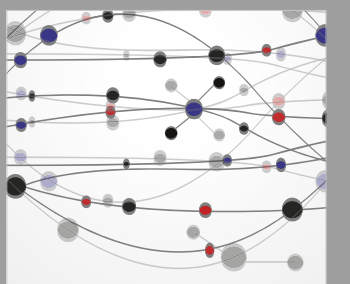

The Scientific World Journal
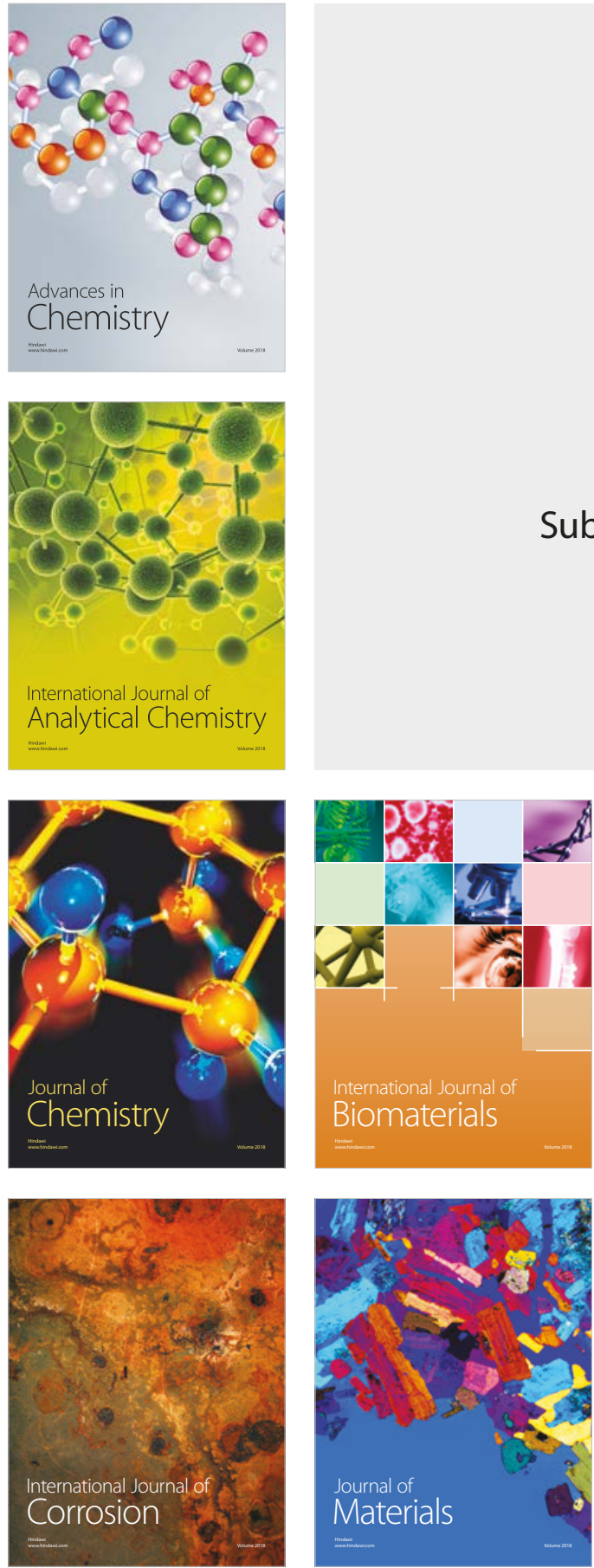

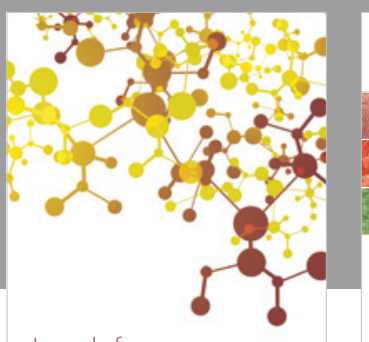

Journal of

Applied Chemistry
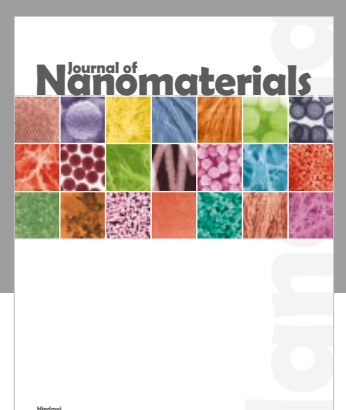

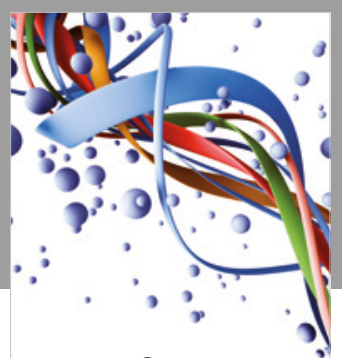

Scientifica

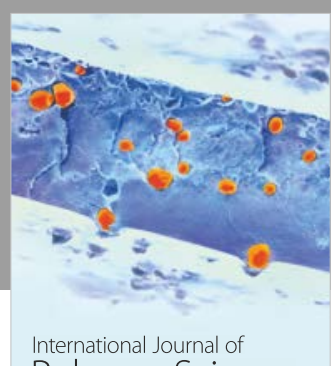

Polymer Science

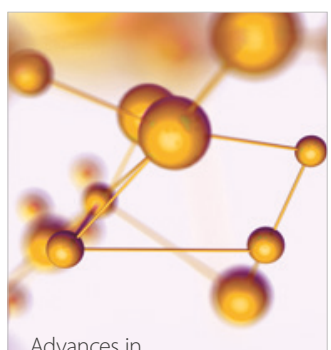

Physical Chemistry
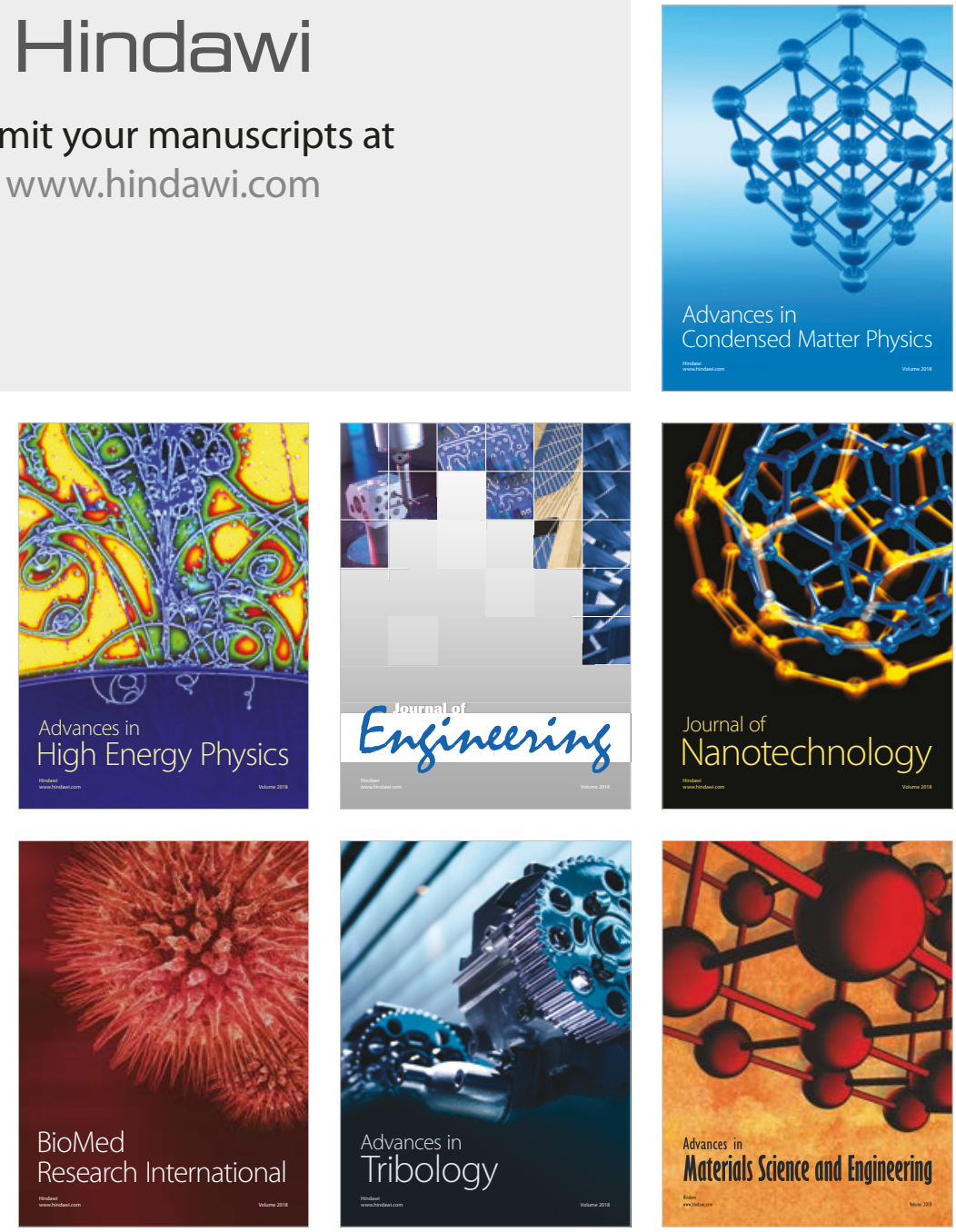